\title{
Optical Properties of One-Dimensional Binary Plasma Dielectric Photonic Crystal
}

\author{
Bin Guo and Mingxiang Gao
}

\begin{abstract}
The optical properties of electromagnetic (EM) waves propagating in one-dimensional plasma dielectric photonic crystal made of alternate thin layers of two materials namely micro plasma layer and dielectric material layer is studied theoretically. The dispersions both for $\omega\left\langle\omega_{p}\right.$ and $\left.\omega\right\rangle$ $\omega_{p}$ are deduced by transfer matrix method and the photonic band gap structure and the reflection spectra are computed. The results show that the band gap structure and reflection spectra are tuned correspondingly due to the dielectric constant of the microplasma layer modified differently in different frequency ranges. Parameter dependence of the effects is calculated and discussed.
\end{abstract}

Index Terms-plasma; dispersion; photonic band structure; reflection spectra

\section{INTRODUCTION}

Photonic crystals (PCs) are composed of periodic dielectric or metal-dielectric nanostructures that affect the propagation of electromagnetic (EM) waves in the same way as the periodic potential in a semiconductor crystal affects the electron motion by defining allowed and forbidden electronic energy bands. Essentially, PCs contain regularly repeating internal regions of high and low dielectric constant. Photons (behaving as waves) propagate through the nanostructures depending - or not - depending on their wavelength. The wavelengths of light that are allowed to travel are known as modes, and the groups of allowed modes form bands. Disallowed bands of wavelengths are called photonic band gaps. Since the pioneering works of Yablonovitch [1] and John [2] on this field many new inquisitive ideas have been developed. The main attraction of the PCs is the existence of forbidden band gaps in their transmission spectra. To achieve suitable band gaps, there are great efforts to obtain tunability of band gaps [3-6]. Recently, a lot of studies on plasma photonic band gaps materials, which physics properties of dielectric constant, can vary continuously in space with the changes of frequency of incidence electromagnetic (EM) waves. The change in the physics properties makes the plasma photonic band gaps structure very different in behavior from the conventional composite materials. Now, these periodically plasma structure is known as plasma photonic crystal (PPC), which is presented by Hojo and Mase [7]. The technologies applications of PPC are now expanding widely as, for example, in plasma lens [8], plasma antenna [9], plasma stealth aircraft [10-13], etc. And the reflectionless transmission due to the Fabry-Perot resonance in PPC can be applied to frequency filters [14] and interferometers [15]. Moreover, PPC has not only the characteristics of photonic crystal, but also the characteristics of plasma. Therefore we can regulate the plasma parameters to achieve the regulation of PPC. Moreover, by replacing solid material with plasma, two important features are added to conventional PC: time-varying controllability and strong dispersion around the electron plasma density. These two features make the frequency of EM wave propagating in PPC range from microwaves to $\mathrm{THz}$ waves, according to the scale and the electron density of plasma [16].

PPC is spatially periodic distribution structure, including plasma and dielectric periodic placed, or PPC is plasma layer with periodic distribution density. Several researches have been analyzed both analytically and numerically. Kuo et al. [17] have studied the interaction of EM waves with rapidly created time-varying periodic plasma. Hojo Hitoshi et al. [7] discussed the dispersion of EM waves in one-dimension PPC using a method analogous to Kroning-Penny's problem in quantum physics. Liu et al. [18, 19] have investigated the EM waves propagating process and reflection and transmission electric field of Gauss pulses passing the PPC. Laxmi et al. [20] have studied the photonic band gap effects in one-dimension plasma dielectric photonic crystal. O. Sakai et al. have studied two-dimension PPC both experimentally [21] and numerically [22].

In the presented communication, we have studied the dispersion and reflection properties of a multi-layered structure with microplasma materials slabs. It has been founded that the plasma parameters have marked influence on their photonic band gap structures and reflection spectra in optical region.

The structure of the paper is organized as follows. The model and corresponding analytical formulas, such as the dispersion relation and the reflection coefficient, are introduced in Sec. II. Numerical results are presented and discussed in Sec.III. Finally, conclusions are given in Sec. IV.

\section{MODEL AND FORMULATIONS}

The schematic diagram of the EM waves propagating in one-dimensional PPC is shown in Fig.1. We assume that the plasma dielectric photonic crystal is divided into a number of units and each unit has two medium which one is plasma materials with width $a$ and the other is background material with width $b$, and $\Lambda=a+b$ is the width of unit sell. For this periodic structure, the dielectric function has the form as given by 


$$
\varepsilon(x)=\left\{\begin{array}{cc}
1-\frac{\omega_{p}^{2}}{\omega^{2}} & n \Lambda<z<n \Lambda+a, \\
& \text { in plasma materials } \\
\varepsilon_{b} & n \Lambda+a<z<(n+1) \Lambda, \\
& \text { in background materials }
\end{array}\right.
$$

where $\omega$ is the frequency of incidence, and $\omega_{p}=\left(n_{e} e^{2} / \varepsilon_{0} m\right)^{1 / 2}$ is plasma frequency, where $n_{e}$ is electron density of plasma layer, $e$ is absolute

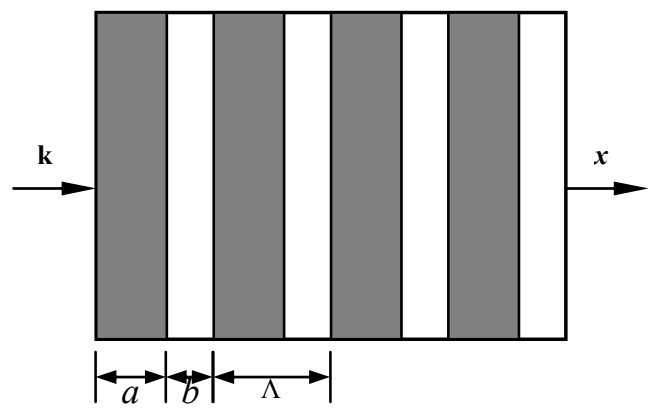

Figure 1. Schematic diagram of normal incident EM wave propagation in 1D PPC.

Electron charge, $m$ is electron mass, $\varepsilon_{0}$ is the permittivity in free space, $\varepsilon_{b}$ is the dielectric constant of the background material, and where the indices $n$ denotes the $n$th unit.

Now the wave equation for EM waves propagating along the $\mathrm{x}$-axis maybe written as

$$
\frac{d^{2} E(x)}{d x^{2}}+\frac{\omega^{2}}{c^{2}} \varepsilon(x) E(x)=0
$$

The electric field $\mathrm{E}(\mathrm{x})$ within each homogeneous layer is a combination of right-traveling waves and left-traveling waves and so it can be expressed as the sum of the incident wave and the reflected wave plane waves. Thus the solution of electric field $\mathrm{E}(\mathrm{x})$ for the nth unit can be written as, for $\omega$ $<\omega \mathrm{p}$

$$
E(z)=\left\{\begin{array}{l}
A_{n} e^{k_{p x}\{x-n \Lambda\}}+\bar{A}_{n} e^{-k_{p x}\{x-n \Lambda\}} \\
B_{n} e^{\mathrm{i} k_{b x}\{x-n \Lambda\}}+\bar{B}_{n} e^{-\mathrm{i} k_{b x}\{x-n \Lambda\}}
\end{array}\right.
$$

and for $\omega>\omega_{p}$

$$
E(z)=\left\{\begin{array}{l}
A_{n} e^{\mathrm{i} k_{p x}^{\prime}\{x-n \Lambda\}}+\bar{A}_{n} e^{-\mathrm{i} k_{p x}^{\prime}\{x-n \Lambda\}} \\
B_{n} e^{\mathrm{i} k_{b x}\{x-n \Lambda\}}+\bar{B}_{n} e^{-\mathrm{i} k_{b x}\{x-n \Lambda\}}
\end{array}\right.
$$

where

$$
\begin{gathered}
k_{b x}=\left(\frac{\omega^{2}}{c^{2}} \varepsilon_{b}\right)^{1 / 2}, k_{p x}=\left[\frac{\omega^{2}}{c^{2}}\left(\frac{\omega_{p}^{2}}{\omega^{2}}-1\right)\right]^{1 / 2}, \\
k_{p x}^{\prime}=\left[\frac{\omega^{2}}{c^{2}}\left(1-\frac{\omega_{p}^{2}}{\omega^{2}}\right)\right]^{1 / 2},
\end{gathered}
$$

where $\boldsymbol{A}_{n}, \bar{A}_{n}$ and $\boldsymbol{B}_{n}, \bar{B}_{n}$ are the amplitudes of counter propagation waves in different unit and $c$ is the speed of light in free space.

For obtaining the optical properties of the proposed structure we are using transfer matrix method. Therefore, the constants $\mathrm{An}, \bar{A}_{n}$ and $\mathrm{Bn}, \bar{B}_{n}$ are related with $2 \times 2$ matrix formulation for a period.

Then we look for the solution the matrix elements for two cases, $\omega<\omega_{p}$ and $\omega>\omega_{p}$.

Firstly, we impose the continuity conditions of $E_{x}$ and $H_{y}$ at the surface $z=(n-1) \Lambda$ and $z=(n-1) \Lambda+b$. We can obtain, for $\omega<\omega_{p}$

$$
\begin{aligned}
& \left(\begin{array}{cc}
1 & 1 \\
1 & -1
\end{array}\right)\left(\begin{array}{l}
A_{n-1} \\
\bar{A}_{n-1}
\end{array}\right)=\left(\begin{array}{cc}
e^{-i k_{b z} \Lambda} & e^{i k_{b z} \Lambda} \\
\frac{\mathrm{i} k_{b z}}{k_{p z}} e^{-i k_{b z} \Lambda} & -\frac{\mathrm{i} k_{b z}}{k_{p z}} e^{i k_{b z} \Lambda}
\end{array}\right)\left(\begin{array}{l}
B_{n} \\
\bar{B}_{n}
\end{array}\right) \\
& \left(\begin{array}{cc}
e^{-\mathrm{i} k_{b z} a} & e^{\mathrm{i} k_{b z} a} \\
e^{-\mathrm{i} k_{b z} a} & -e^{\mathrm{i} k_{b z} a}
\end{array}\right)\left(\begin{array}{l}
B_{n} \\
\bar{B}_{n}
\end{array}\right)=\left(\begin{array}{cc}
e^{-k_{p z} a} & e^{k_{p z} a} \\
\frac{k_{p z}}{i k_{b z}} e^{-k_{p z} a} & -\frac{k_{p z}}{i k_{b z}} e^{k_{p z} a}
\end{array}\right)\left(\begin{array}{l}
A_{n} \\
\bar{A}_{n}
\end{array}\right)
\end{aligned}
$$

and for $\omega>\omega_{p}$

$$
\begin{aligned}
& \left(\begin{array}{cc}
1 & 1 \\
1 & -1
\end{array}\right)\left(\begin{array}{l}
A_{n-1} \\
\bar{A}_{n-1}
\end{array}\right)=\left(\begin{array}{cc}
e^{-\mathrm{i} k_{b z} \Lambda} & e^{\mathrm{i} k_{b z} \Lambda} \\
\frac{k_{b z}}{k_{p z}^{\prime}} e^{-\mathrm{i} k_{b z} \Lambda} & -\frac{k_{b z}}{k_{p z}^{\prime}} e^{\mathrm{i} k_{b z} \Lambda}
\end{array}\right)\left(\begin{array}{l}
B_{n} \\
\bar{B}_{n}
\end{array}\right) \\
& \left(\begin{array}{cc}
e^{-\mathrm{i} k_{b z} a} & e^{\mathrm{i} k_{b z} a} \\
e^{-\mathrm{i} k_{b z} a} & -e^{\mathrm{i} k_{b z} a}
\end{array}\right)\left(\begin{array}{l}
B_{n} \\
\bar{B}_{n}
\end{array}\right)=\left(\begin{array}{cc}
e^{-\mathrm{i} k_{p z}^{\prime} a} & e^{\mathrm{i} k_{p z}^{\prime} a} \\
\frac{k_{p z}^{\prime}}{k_{b z}} e^{-\mathrm{i} k_{p z}^{\prime} a} & -\frac{k_{p z}^{\prime}}{k_{b z}} e^{\mathrm{i} k_{p z}^{\prime} a}
\end{array}\right)\left(\begin{array}{l}
A_{n} \\
\bar{A}_{n}
\end{array}\right)
\end{aligned}
$$

From equations (4a) and (4b) eliminating $\left(\begin{array}{c}B_{l} \\ \bar{B}_{l}\end{array}\right)$, we can obtain

$$
\left(\begin{array}{l}
A_{n-1} \\
\bar{A}_{n-1}
\end{array}\right)=\left(\begin{array}{ll}
M_{11} & M_{12} \\
M_{21} & M_{22}
\end{array}\right)\left(\begin{array}{l}
A_{n} \\
\bar{A}_{n}
\end{array}\right)
$$

where $\left(\begin{array}{ll}M_{11} & M_{12} \\ M_{21} & M_{22}\end{array}\right)$ is unite transfer matrix, and we can obtain the element of the matrix from equations (4a), (4b) and (5) with the condition $M_{11} M_{22}-M_{12} M_{21}=1$, The matrix elements $M_{11}, M_{12}, M_{21}$ and $M_{22}$ are obtain as, for $\omega<\omega_{p}$

$$
\begin{gathered}
M_{11}=\left[\cos k_{b x} b-\frac{1}{2} \mathrm{i}\left(\frac{\mathrm{i} k_{b x}}{k_{p x}}+\frac{k_{p x}}{\mathrm{i} k_{b x}}\right) \sin k_{b x} b\right] e^{-k_{p x} a} \\
M_{12}=\left[-\frac{1}{2} \mathrm{i}\left(\frac{\mathrm{i} k_{b x}}{k_{p x}}-\frac{k_{p x}}{\mathrm{i} k_{b x}}\right) \sin k_{b x} b\right] e^{k_{p x} a} \\
M_{21}=\left[\frac{1}{2} \mathrm{i}\left(\frac{\mathrm{i} k_{b x}}{k_{p x}}-\frac{k_{p x}}{\mathrm{i} k_{b x}}\right) \sin k_{b x} b\right] e^{-k_{p x} a} \\
M_{22}=\left[\cos k_{b x} b+\frac{1}{2} \mathrm{i}\left(\frac{\mathrm{i} k_{b x}}{k_{p x}}+\frac{k_{p x}}{\mathrm{i} k_{b x}}\right) \sin k_{b x} b\right] e^{k_{p x} a}
\end{gathered}
$$

and for $\omega>\omega_{p}$

$$
\begin{gathered}
M_{11}=\left[\cos k_{b x} b+\frac{1}{2} \mathrm{i}\left(\frac{k_{b x}}{k_{p x}^{\prime}}+\frac{k_{p x}^{\prime}}{k_{b x}}\right) \sin k_{b x} b\right] e^{-\mathrm{i} k_{p x}^{\prime} a} \\
M_{12}=\left[-\frac{1}{2} \mathrm{i}\left(\frac{k_{b x}}{k_{p x}^{\prime}}-\frac{k_{p x}^{\prime}}{k_{b x}}\right) \sin k_{b x} b\right] e^{\mathrm{i} k_{p x}^{\prime} a} \\
M_{21}=\left[\frac{1}{2} \mathrm{i}\left(\frac{k_{b x}}{k_{p x}^{\prime}}-\frac{k_{p x}^{\prime}}{k_{b x}}\right) \sin k_{b x} b\right] e^{-\mathrm{i} k_{p x}^{\prime} a} \\
M_{22}=\left[\cos k_{b x} b+\frac{1}{2} \mathrm{i}\left(\frac{k_{b x}}{k_{p x}^{\prime}}+\frac{k_{p x}^{\prime}}{k_{b x}}\right) \sin k_{b x} b\right] e^{\mathrm{i} k_{p x}^{\prime} a}
\end{gathered}
$$

Then, from the periodicity of $\varepsilon(\mathrm{x})$ shown by equation (1) and also of given by $\mathrm{E}(\mathrm{x})=\mathrm{E}(\mathrm{x}+\Lambda)$, and using the Floquet theorem [23], we can obtain that the phase coefficient e-iK $\Lambda$ is the eigenvalue of the eigenequation which can be 
described as below

$$
\left(\begin{array}{cc}
M_{11}-e^{-\mathrm{i} K \Lambda} & M_{12} \\
M_{21} & M_{22}-e^{-\mathrm{i} K \Lambda}
\end{array}\right)=0
$$

Here, $K$ is known as Bloch wave number and is a function of $\omega$. Then the dispersion relation can be obtained from the equation (6), as written by

$$
K(\omega)=\frac{1}{\Lambda} \cos ^{-1}\left[\frac{1}{2}\left(M_{11}+M_{22}\right)\right]
$$

Substituting equations (6a) and (6b) in equation (8), we can obtain the dispersion relation of $\mathrm{P}$-polarization electromagnetic waves propagation in one-dimension plasma dielectric photonic crystal, for $\omega<\omega_{p}$,

$$
\cos K \Lambda=\cosh k_{p z} a \cos k_{b z} b+\frac{1}{2}\left(\frac{k_{p z}}{k_{b z}}-\frac{k_{b z}}{k_{p z}}\right) \sinh k_{p z} a \sin k_{b z} b
$$

and for $\omega>\omega_{p}$

$$
\cos K \Lambda=\cos k_{p z}^{\prime} a \cos k_{b z} b-\frac{1}{2}\left(\frac{k_{p z}^{\prime}}{k_{b z}}+\frac{k_{b z}}{k_{p z}^{\prime}}\right) \sin k_{p z}^{\prime} a \sin k_{b z} b
$$

The reflection coefficient of such considered periodic structure for $N$ number of period can be obtained by using Eqs.(4). The reflection $r_{N}=\left(\frac{\bar{A}_{0}}{A_{0}}\right)_{\bar{A}_{N}=0}$ when the reflection by $N$ th layer is zero. So, the reflectance of the proposed structure for $N$ number of period is given by [24]

$$
R=\left|r_{N}\right|^{2}=\frac{\left|M_{21}\right|^{2}}{\left|M_{21}\right|^{2}+\left(\frac{\sin (K \Lambda)}{\sin (N K \Lambda)}\right)^{2}}
$$

So, we can use the Eqs.(9) and (10) to obtain and discuss the dispersion and reflection of the proposed structure.

\section{RESULTS AND DisCUSSION}

To evaluate the optical properties, band gap structure and reflectance, of the proposed multi-layered we used the Eqs. (9) and (10). We note that there are there selective parameters, $\omega \mathrm{p} \Lambda / 2 \pi \mathrm{c}$ (For simplicity, we introduce the dimensions variable $\omega \Lambda / 2 \pi \mathrm{c}$ which is normalized to 1 at $\omega$ $=\omega \mathrm{p}$ ), plasma filling factor $\mathrm{f}$ (which is expressed as $\mathrm{f}=$ $\mathrm{a} / \Lambda$ ), and the dielectric constant value $\varepsilon \mathrm{b}$ which is normalized to $\varepsilon b / \varepsilon 0$ in the numerical calculations.

Firstly the dispersion relation for $\omega_{p} \Lambda / 2 \pi c=1, \varepsilon_{b}=1$ (for air), $f=0.1$ and $f=0.3$ is shown in Fig. 2, of which (a) depicts
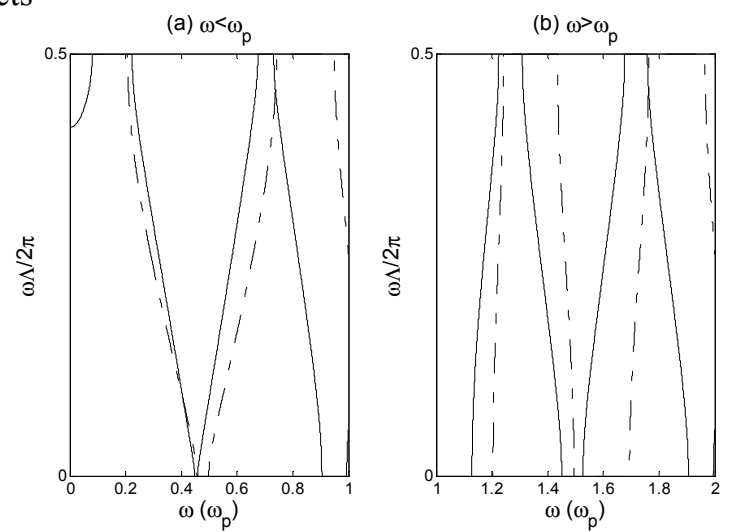

Figure 2. Photonic band gap structures for $\omega \mathrm{p} \Lambda / 2 \pi \mathrm{c}=1$, $\varepsilon b=1$ (for air), $f=0.1$ (solid line) and $\mathrm{f}=0.3$ (solid dot line)
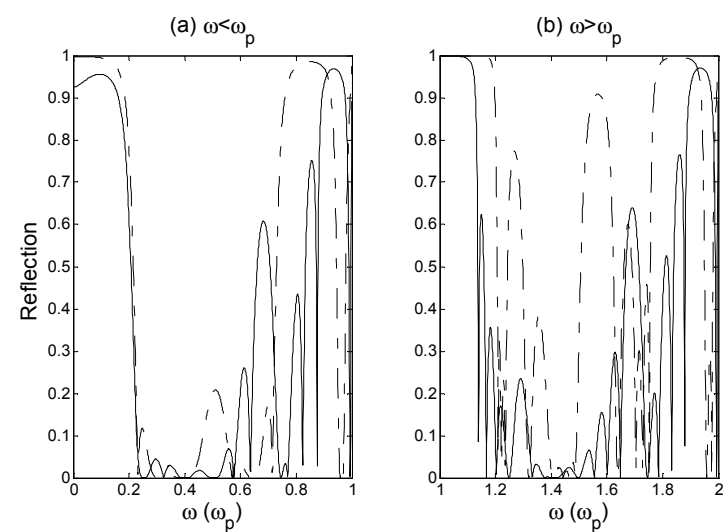

Figure 3. The reflection spectra versus the frequency of normal incident EM wave, the parameters are the same as Fig.2.

The dispersion relation of $\omega<\omega \mathrm{p}$ and (b) does the one of $\omega>\omega$ p. It can be seen that the dispersion relation becomes a band structure with frequency with frequency gaps, and there is a cut-off frequency. It is clear that the frequency band gap becomes larger with the increase in plasma filling factor. Fig. 3 shows the reflection spectra versus the frequency of incident EM wave. Here we have taken the number of periods, $\mathrm{N}=4$, which is fixed in the numerical calculation. Other parameters are as the same as above. From Fig.3, we can easily conclude that the photonic band gap is getting larger with an increase in the plasma filling factor. The gap region shifts towards the higher frequency with the increase in plasma filling factor.
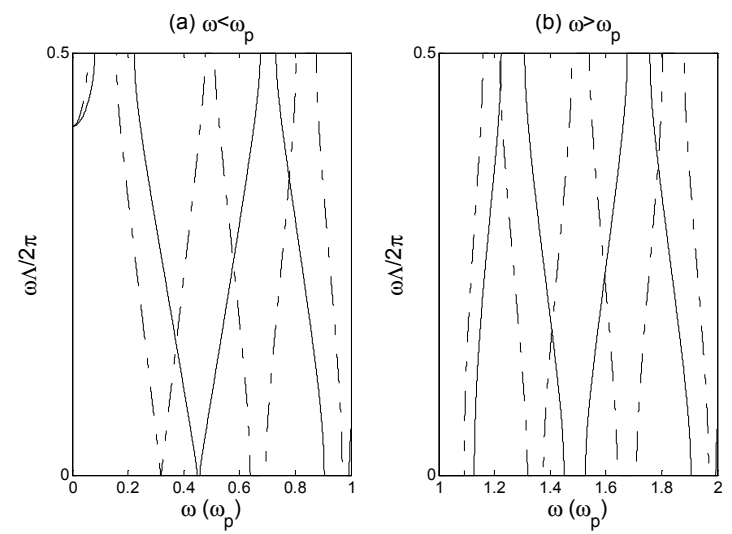

Figure 4. Photonic band gap structures for $\omega \mathrm{p} \Lambda / 2 \pi \mathrm{c}=1, \mathrm{f}=0.1$,
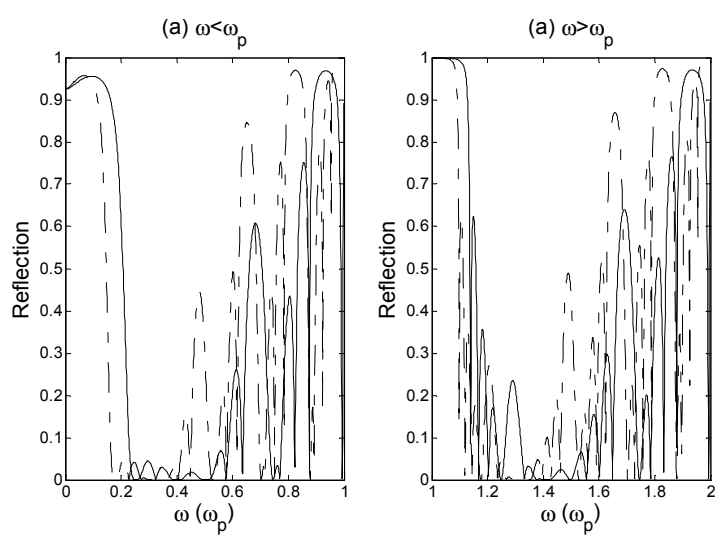

Figure 5. The reflection spectra versus the frequency of normal incident EM wave, the parameters are the same as Fig.4

Next the dispersion relation for $\omega \mathrm{p} \Lambda / 2 \pi \mathrm{c}=1, \mathrm{f}=0.1, \mathrm{cb}=$ 1 (for air), and $\varepsilon b=2$ (for $\mathrm{SiO} 2$ ) is shown in Fig 4, where the 
frequency band gap is smaller for the larger dielectric constant value $\varepsilon b$ of background material or increasing the background material dielectric constant value $\mathrm{eb}$ leads to the creation of new photonic band gaps and to flattering of the photonic band gaps which means the reduction of group velocity. Fig. 5 shows the reflection spectra versus the frequency of incident EM wave. It is obvious from Fig.5 that the number of frequency band gap becomes larger and the frequency band gap gets flatter and deeper appreciably increasing the background material dielectric constant $\varepsilon b$. At the same time, the frequency bandwidths will be broadened and the space between the photonic band gaps will be reduced. Comparing with the Fig.2 and Fig.3 or Fig. 4 and Fig.5, there are highly consistent with each other for depiction of the photonic band gap.

\section{CONCLUSION}

In summary, we have deduced the dispersion relations of EM wave propagation in one dimensional multi-layered structures containing plasma material both for $\omega<\omega_{p}$ and $\omega>\omega_{p}$, exclusively. The graph for dispersion relations and the reflection spectra is computed and plotted. The effects of plasma parameters such as plasma filling factor and the background material dielectric constant value are discussed. The results show that the proposed multi-layered structure can be acted as a tunable photonic crystal which can be controlled by the external parameters. Comparing with conventional photonic crystal, the multi-layered structure containing plasma material, as an innovative and dynamic field, will bring more new physics phenomena, draw more attention and lead to many new applications in the future.

\section{ACKNOWLEDGMENT}

This work was supported by National Natural Science Foundation of China (NNSFC) under grant No.10747153 and the Fundamental Research Funds for the Central Universities.

\section{REFERENCES}

[1] E. Yablonovitch, "Inhibited Spontaneous Emission in Solid-State Physics and Electronics," Phys. Rev. Lett., vol. 58, May 1987, pp.2059-2062

[2] S. John, "Strong localization of photons in certain disordered dielectric superlattices," Phys. Rev. Lett., vol. 58, May 1987, pp.2486-2489

[3] Busch K and John S, "Liquid-Crystal Photonic-Band-Gap Materials: The Tunable Electromagnetic Vacuum," Phys. Rev. Lett., vol. 83, Aug. 1999, pp.967-970

[4] Meng Q B, Fu C H, Hayami S, Gu Z Z and Sato O, "Effects of external electric field upon the photonic band structure in synthetic opal infiltrated with liquid crystal," J. Appl. Phys., vol. 89, May 2001, pp.5794-5796

[5] Kang D, Maclennan J E, Clar N A, Zakhidov A A and Baughman R $\mathrm{H}$, "Electro-optic Behavior of Liquid-Crystal-Filled Silica Opal
Photonic Crystals: Effect of Liquid-Crystal Alignment," Phys. Rev. Lett., vol. 86, Apr. 2001, pp.4052-4505

[6] J. M. Lourtioz, H. Benisty, V. Berger, J. M. Gérard, G. Maystre, and A. Techelnokov. Photonic Crystals: Towards Nanoscale Photonic Devices, Springer-Verlag Berlin Heidelberg, 2005

[7] H. Hojo, A. Mase, "Dispersion Relation of Electromagnetic Waves in One-Dimensional Plasma Photonic Crystals," J. Plasma Fusion Res., vol. 80, Jan. 2004, pp.89-90

[8] A. A. Goncharov, A. N. Dobrovolsky, A. V. Zatuagan, and I. M. Protsenko, "High-current plasma lens," IEEE Trans. Plasma Sci., Vol. 21, no. 5, pp. 573-577, Oct. 1993.

[9] T. J. Dwyer, J. Greig, D. Murphy, J. Perin, R. Pechaceket, and M. Raleigh, "On the feasibility of using an atmospheric discharge plasma as RF antenna," IEEE Trans. Antenna and Propag., vol. AP-32, no. 2, pp. 141-146, Feb. 1984.

[10] R. J. Vidmar, "On the use of atmospheric pressure plasmas as electromagnetic reflectors and absorbers," IEEE Trans. Plasma Sci., vol. 18, no. 4, pp. 733-741, Aug. 1990.

[11] D. L. Tang, A. P. Sun, X. M. Qiu, and Paul K. Chu, "Interaction of electromagnetic waves with a magnetized nouniform plasma slab," IEEE Trans. Plasma Sci., vol. 31, no. 3, pp. 405-410, June. 2003.

[12] B. Guo, and X. G. Wang, "Power absorption of high-frequency electromagnetic waves in a partially ionized magnetized plasma," Phys. Plasmas, vol. 12, no. 8, pp. 084506-1-4, Aug. 2005.

[13] B. Guo, X. G. Wang, and Y. Zhang, "FDTD numerical simulation of microwaves in an unmagnetized atmosphere plasma," Plasma Sci. Technol., vol. 8, no. 5, pp. 558-560, Sept. 2006.

[14] Pacher C, Rauch C, Strasser G, Gornik E, Elsholz F, Wacker A, Kihblich G, and Scholl E, Antireflection coating for miniband transport and Fabry-Perot resonance in GaAs/AlGaAs superlattice, Appl. Phys. Lett., vol. 79, Sep. 2001, pp.1486-1487

[15] Waters R L, Aklufi M E, Macromachined Fabry-Perot interfermeter motion detection, Appl. Phys. Lett., vol. 81, Oct. 2002, pp.3320-3322

[16] O. Sakai, T. Sakaguchi, and K. Tachibana, "Plasma photonic crystals in two-dimensional arrays of microplasmas," Contrib. Plasma Phys., vol. 47, no. 1/2, pp. 96-102, Jan. 2007.

[17] Kuo S P, James Fatith., "Interaction of an electromagnetic wave with a rapidly created spatially periodic plasma," Phys. Rev. E, vol. 56, Aug. 1997, pp.2143-2150

[18] Liu S B, Zhu C X, Yuan N C, "FDTD simulation for plasma photonic crystals," Acta Physica Sinica., vol. 54, June. 2005, pp.2804-2809

[19] Liu S B, Gu C Q, Zhou J J and Yuan N C, "FDTD simulation for magnetized plasma photonic crystals," Acta Physica Sinica., vol. 55, March 2006, pp.1283-1288

[20] Laxmi S, Parmanand M, "Photonic band gap effect in one-dimensional plasma dielectric photonic crystals," Solid State Communications, vol.138, Apr. 2006, pp.160-164

[21] O. Sakai, T. Sakaguchi and K. Tachibana, "Verification of a plasma photonic crystal for microwaves of millimeter wavelength range using two-dimensional array of columnar microplasmas," Appl. Phys. Lett., vol. 87 , Dec. 2005, pp.241505-03

[22] O. Sakai, K. Tachibana, "Properties of Electromagnetic Wave Propagation Emerging in 2-D Periodic Plasma Structures," IEEE Trans. Plasma Sci., vol. 35, Oct. 2005, pp.1267-1273

[23] Magnus, W. and Winkler, S, Floquet's Theorem, New York: Dover, pp. $3-8,1979$.

[24] J.M. Bendickson, J.P. Dowling and M. Scalora, "Analytic expressions for the electromagnetic mode density in finite, one-dimensional, photonic band-gap structures," Phys. Rev. E,vol.53, Apr.1996, pp.4107-4151

Bin Guo, was born in Hubei, China, in 1978. He received the B. S., M. S and $\mathrm{Ph}$. D degree from the Dalian University of Technology, Liaoning, China, in 2000, 2002 and 2006, respectively. He is currently an Associate Professor with the Wuhan University of Technology, from 2006. His research interests include quantum optics, photonic crystal, electromagnetci propagation and microwave technique. 